\title{
Is the cultural evolution of technology cumulative or combinatorial?
}

\author{
James Winters*1,2 \\ ${ }^{1}$ Collective Computation Group, School of Collective Intelligence, UM6P, Morocco \\ ${ }^{2}$ Mint Group, Max Planck Institute for the Science of Human History, Germany
}

September 25, 2020

\begin{abstract}
Explanations of human technology often point to both its cumulative and combinatorial character. Using a novel computational framework, where individual agents attempt to solve problems by modifying, combining and transmitting technologies in an open-ended search space, this paper re-evaluates two prominent explanations for the cultural evolution of technology: that humans are equipped with (i) social learning mechanisms for minimizing information loss during transmission, and (ii) creative mechanisms for generating novel technologies via combinatorial innovation. Here, both information loss and combinatorial innovation are introduced as parameters in the model, and then manipulated to approximate situations where technological evolution is either more cumulative or combinatorial. Compared to existing models, which tend to marginalize the role of purposeful problem-solving, this approach allows for indefinite growth in complexity while directly simulating constraints from history and computation. The findings show that minimizing information loss is only required when the dynamics are strongly cumulative and characterised by incremental innovation. Contrary to previous findings, when agents are equipped with a capacity for combinatorial innovation, low levels of information loss are neither necessary nor sufficient for populations to solve increasingly complex problems. Instead, higher levels of information loss are advantageous for unmasking the potential for combinatorial innovation. This points to a parsimonious explanation for the cultural evolution of technology without invoking separate mechanisms of stability and creativity.
\end{abstract}

\section{Introduction}

Over the last 60, 000 years, humans went from a species which relied on stone tools and lived in small groups to one that builds microprocessors and occupies cities. Any theory of human technology, which seeks to understand and explain this unparalleled change, must account for three general observations. First, all human technology serves an underlying purpose: to solve problems 1]. This problem-solving nature of technology is equally true of travelling to the Moon with a Saturn V rocket as it is of using a pencil to take notes. Second, human technology is combinatorial, wherein a finite number of existing technologies are combined to generate novel ones [1 1 . Hafting a projectile point onto a spear, reusing iron door hinges as ship rudders, and affixing wheels to a luggage

\footnotetext{
*james.winters@um6p.ma
} 
case all qualify as examples of combination. Finally, technological change is to is to some extent cumulative [6 11], in that previous iterations of technologies serve as the foundation for subsequent innovations. The steam engine did not spontaneously arrive in the $18^{\text {th }}$ Century; rather, it was the culmination of refining, combining, and improving existing technologies that date back to at least the $1^{\text {st }}$ Century 12 .

No existing model of technological evolution adequately addresses all three observations within a single framework. Here I attempt a first pass at formulating such a theory using agent-based modelling. Formal and computational models figure prominently in the cultural evolution literature and have produced several important theoretical insights. As it currently stands, much of this literature is centered around the issue of stability 13 15], and whether high fidelity transmission is necessary for cumulative dynamics 16 19]. Less attention is paid to the creative aspects of production 5, 20 23, with only a small number of models considering cumulative growth in the context of combinatorial innovation 17,24,26. Furthermore, while many theoretical accounts acknowledge the role of technologies as problem-solving devices, cultural evolutionary models tend to deal with this relationship in one of two ways: either by reducing the effects of problem solving to an intrinsic feature of a technology (using a payoff function [17]) or by ignoring the issue altogether [23. This misses a key characteristic of problem-solving: that technologies are not only copied, modified and combined to solve existing problems, they also continually open up new problem-solving opportunities for exploration [4]. Consider the wheel, which today is an indispensable component of numerous technologies, yet many of these modern functions are only tenuously connected to its original role as a Potter's wheel in Ancient Mesopotamia.

Two consequences follow from a fuller appreciation of the relationship between technological evolution and problem-solving. First, the cultural evolution of technology is a path-dependent process, wherein choices made at one point in time can subsequently bias the emergence of future states (historical constraint). As such, due to what is known as historical lock-in [27], path-dependency can inhibit the discovery of more efficient outcomes (especially if a solution is good enough at solving an existing set of problems). The reason why I type on a QWERTY keyboard is largely the result of a historical design solution for the problem of jamming on mechanical typewriters. Second, the time and resource costs associated with problem-solving scale with the complexity of the problems 28 30] (computational constraint). As problems increase in complexity, so too do the computational resources needed to maintain long-term improvements. Intuitively, landing a person on the moon is orders of magnitude more difficult than flying across a field, and it is probably why achieving the latter preceded the former by 66 years. This leaves us with the following puzzle: How is it that human technology is capable of solving increasingly complex problems when faced with constraints from history and computation?

The current literature points to two possible answers to this question. The first possibility focuses on the stability of technological lineages and assumes there exists preservative mechanisms or processes for mitigating information loss; be it inductive biases for filling in inferential gaps [15], specialised adaptations for high fidelity social learning [9, 31], or demographic factors that minimise sampling error 13. Under this perspective, mechanisms for preserving information provide a stable platform for populations to cumulatively build upon the cultural and technical know-how of previous generations. This lowers the computational load as individuals at the next generation (literally) do not need to reinvent the wheel. The second possibility is found in the combinatorial nature of human technology. Models of combinatorial evolution highlight the generative aspect of human culture and technology 1, 20, 32, 33. By creating links between technologies located in distant regions of the search space, combination provides a simple and compelling mechanistic explanation for rapid and radical bursts of innovation often witnessed in the history of technology 25, 34. Without such mechanisms, high levels of information loss are predicted to impede the formation of shared and 
enduring traditions 10,18, whereas the absence of combination is expected to confine innovation to local searches and inhibit exploration of adjacent possible technologies [1, 33.

To investigate these claims, this paper introduces a model of cultural evolution in which individuals solve problems in an open-ended, procedurally generated search space. Building on previous work, where movement through this space is modulated by the efficiency with which problems are solved, cultural evolutionary dynamics are represented here as search processes over the space of problems and solutions [35. The goal of individuals in this model is to try and find efficient solutions via two causal routes: optimization (where technologies are refined and optimized towards solving specific problems) and exploration (where technologies are co-opted and repurposed towards solving novel problems). Using this general framework, the aim of the model is to re-evaluate the relative roles of cumulative and combinatorial processes in driving long-term problem-solving. In particular, the model introduces two parameters that feature prominently in the literature on the cultural evolution of technology: the type of innovation $(\alpha)$ and the level of information loss $(\epsilon)$. The type of innovation focuses on the generative aspect of technology and refers to whether innovations are generated by local modifications to existing technologies or through the creation of novel composites. Meanwhile, the level of information loss focuses on the cultural transmission of technologies, and deals with the extent to which these technologies are inherited from one generation to the next.

The results of the model point to three general conclusions. First, mitigating information loss is only advantageous when technological change is purely cumulative and driven by local innovation. Importantly, such change is subject to diminishing returns in the long run, with populations increasingly struggling to solve more complex problems. This leads to the second finding: that some information loss is required to unmask the potential of combinatorial innovation to generate novel solutions and solve increasingly difficult problems. Moreover, simulation runs with combinatorial innovation reach far higher levels of problem complexity, as these runs erase inertia from path-dependency and open up the possibility of exploring distant regions of the search space. Finally, if there is no inheritance of technologies, then runs with local innovation fail to exhibit any growth while runs with combinatorial innovation eventually reach a complexity ceiling (due to the computational costs of having to search for solutions from scratch at each generation).

\section{Model Description}

Cultural evolutionary dynamics are modelled here as search processes over the space of problems and solutions [36]. Problems $(\psi)$ correspond to the specific functional challenges faced by individuals, and solutions $(\mu)$ represent the skills, techniques, or artefacts used to solve these problems (i.e., the application of technologies). Both problems and solutions are operationalized as two-state $[A, B]$ strings of $N$-length. Individuals start out by solving problems in a space of just four possibilities $2^{2}$, but this space has the potential to grow exponentially and theoretically the number of possibilities is unbounded. Crucially, the shape of the search space connecting problems to solutions is an emergent property of the search process: the projection of one point in the solution space onto another point in the problem space, and the distance between these two points, approximates the efficiency of a technology.

A distinct advantage of this approach is that it aims to directly link cultural evolutionary dynamics with constraints from problem-solving. This affords a (potentially) indefinite search: individuals can move between problems of any difficulty within the constraints imposed by the topology of the space and the underlying cultural evolutionary dynamics. In particular, modelling problem-solving as a search process captures the following topological properties: that different problems require different solutions (a knife used for cutting meat will struggle when applied to reinforced concrete); 
that some problems are harder to solve than others (cutting through reinforced concrete is orders of magnitude more difficult than slicing a steak); and that all problems are interconnected to one another with varying degrees (types of cutting are related to one another in that they need to induce a certain level of shear stress).

Model runs take place over $g$ generations and every generation comprised of 10 time-steps. A single run is initialised with a fixed population of individual agents $(K=100)$. For each run, the initial problems and solutions of individuals are randomly generated, with a uniform probability for problems of length $\ell(\psi)=2$ and solutions in the range $\ell(\mu)=[2,4]$. An individual agent can only store a maximum of four solutions at any time-step. These solutions are sorted on the basis of how efficiency they solve the target problem $\left(\psi_{\text {existing }}\right)$; the most efficient being stored as the current solution $\left(\mu_{\text {current }}\right)$. At a given time-step, an agent $k$ attempts to solve problems via three possibilities (subject to the type of innovation parameter, $\alpha$ ): memory, local innovation, and combinatorial innovation. Following 10 time-steps, each agent searches for a novel problem to solve in the search space, with this search process only halting once an agent finds a problem longer than their current solution. In the next generation, all individual agents are replaced by naïve offspring and this new generation inherits the current problem and solutions of their parents (subject to the information loss parameter, $\epsilon$ ).

\subsection{Efficiency $L D[\psi, \mu]$}

Problem solving is represented as the extent to which a solution efficiently maps onto a problem. For instance, when attempting to solve the problem $A B A A$, the solution $A B A B$ is a more efficient fit than $B B B A$. To determine the efficiency with which a cultural trait solves a specific problem, individuals receive a payoff value based on the Levenshtein distance (henceforth, LD) [37]:

$$
L D[\psi, \mu](i, j)= \begin{cases}\max (i, j) & \text { if } \min (i, j)=0 \\
\min \left\{\begin{array}{l}
L D[\psi, \mu](i-1, j)+1 \\
L D[\psi, \mu](i, j-1)+1 \\
L D[\psi, \mu](i-1, j-1)+1_{\psi_{i} \neq \mu_{j}}
\end{array}\right. & \text { otherwise. }\end{cases}
$$

where $L D[\psi, \mu](i, j)$ is the distance between the $i$ th element of problem $\psi$ and the $j$ th element of solution $\mu$. This tells us the minimum number of single-element edits (insertions, deletions, or substitutions) required to transform the solution into the problem. Fewer transformations between a problem and a solution acts as a proxy for more efficient technologies. As such, an optimally efficient technology is one where a problem and its solution are identical, and thus require no transformations.

\subsection{Optimization $\alpha$}

At each time-step, individual agents find efficient outcomes via three routes: memory (an array of stored solutions), local innovation (where an agent takes their current solution and performs the most efficient single-edit modification), and combinatorial innovation (where an agent searches all possible permutations in memory and returns the most efficient outcome). By comparing how each potential solution solves a specific target problem, $\psi_{\text {existing, }}$, an individual chooses the most efficient option and assigns it to memory as their current solution, $\mu_{\text {current }}$ :

$$
\mu_{\text {current }}= \begin{cases}\mu_{\text {novel }}, & \text { if } L D\left[\psi_{\text {existing }}, \mu_{\text {current }}\right]>L D\left[\psi_{\text {existing }}, \mu_{\text {novel }}\right] \\ \mu_{\text {current }}, & \text { otherwise }\end{cases}
$$


where $L D[\psi, \mu]$ is the Levenshtein distance between a problem and a solution. Here, $\mu_{\text {current }}$ is the most efficient solution currently stored in memory, and $\mu_{\text {novel }}$ refers to the most efficient novel solution generated by either combinatorial or local innovation. The extent to which individuals have access to these types of innovation is determined by the parameter $\alpha$ (see Fig. 1). In this model, the following parameter values were considered: $\alpha=[-1.0,0.0,1.0]$. When $\alpha=0.0$, agents generate potential solutions via both local and combinatorial innovation. Agents only generate potential solutions via combinatorial innovation when $\alpha=-1.0$, whereas agents solely rely on local innovation when $\alpha=1.0$.
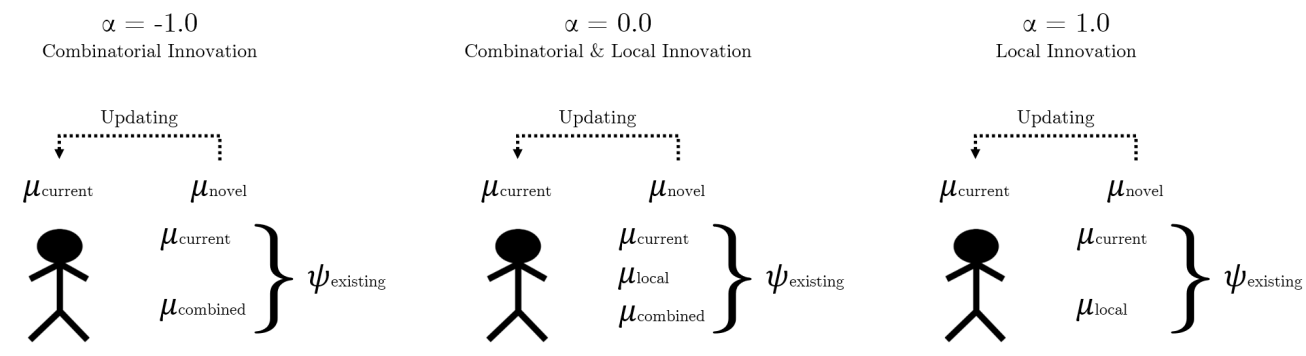

Figure 1: The optimization process at a single time-step for combinatorial innovation $(\alpha=-1.0)$, local innovation $(\alpha=1.0)$, and both combinatorial and local innovation $(\alpha=0.0)$. Here, an individual first generates a set of possible solutions, which, depending on $\alpha$, is either from memory $\left(\mu_{\text {current }}\right)$, local innovation $\left(\mu_{\text {local }}\right)$ and/or combinatorial innovation $\left(\mu_{\text {combined }}\right)$. Each of these solutions is compared with one another in terms of how efficiently they solve the existing input problem $\psi_{\text {existing. }}$. The most efficient solution is then stored memory and becomes the current solution $\left(\mu_{\text {current }}\right)$ at the next time-step.

\subsection{Exploration $\ell(\psi)$}

Following 10 time-steps, individuals decide whether to relocate from an existing problem to a novel one (see Fig. 2). Searching for novel problems, $\psi_{\text {novel }}$, is biased so that agents seek out problems longer than their currently stored solution:

$$
\psi_{\text {existing }}= \begin{cases}\psi_{\text {novel }}, & \text { while } \ell\left(\psi_{\text {existing }}\right) \leq \ell\left(\mu_{\text {current }}\right) \\ \psi_{\text {existing }}, & \text { otherwise }\end{cases}
$$

where $\ell\left(\psi_{\text {novel }}\right)$ is the length of a novel problem in the search process and $\ell\left(\mu_{\text {current }}\right)$ is the length of the current solution. Each iteration of the search is restricted in that agents can only consider neighbouring problems: those which are single edit distance away from the existing problem, $\psi_{\text {existing. }}$. A novel problem, $\psi_{\text {novel }}$, can therefore be longer, shorter, or of the same length as $\psi_{\text {existing. However, }}$. the search only halts once an agent finds a novel problem longer than the current solution, at which point this novel problem becomes the existing one. 


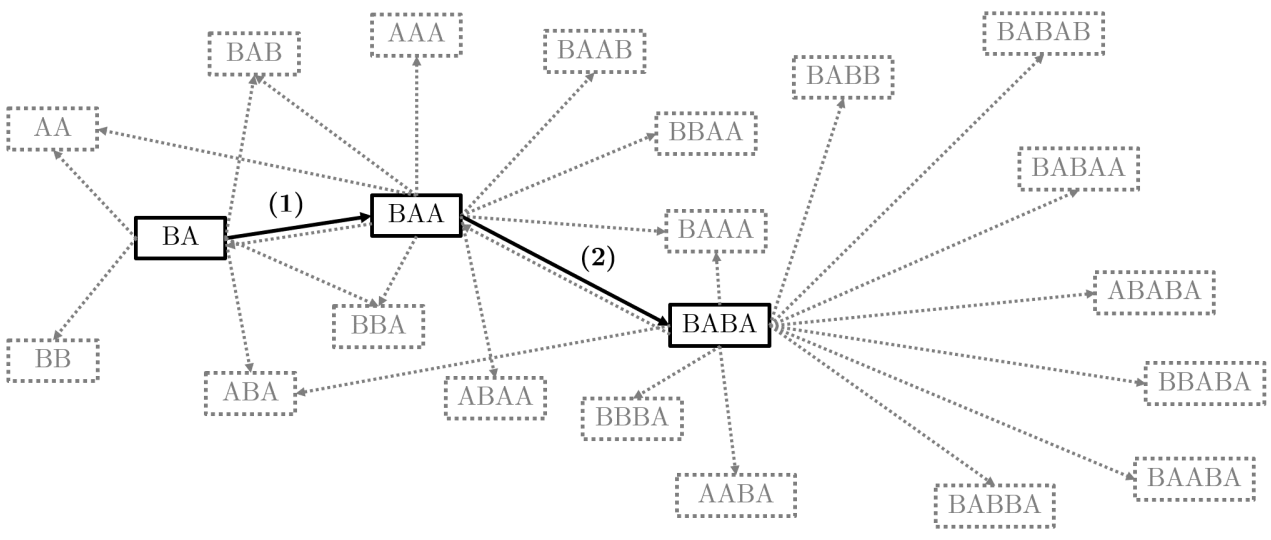

Figure 2: Movement through a small portion of the problem space. Black lines and boxes denote the movement of a single individual (with arrows corresponding to the direction of movement and numbers in brackets representing the order of this movement). Grey directional lines and boxes convey the space of possible problems an individual could move to given their current problem. Here, an individual starts at the problem $B A$, and then moves through the space from problem to problem until they reach $B A B A$. When an individual first moves to the problem $B A A$, the search space grows by a factor of $2^{3}$, and will continue to expand indefinitely by $2^{n}$ so long as individuals are capable of moving to longer problems.

\subsection{Inheritance $\epsilon$}

At the start of each generation, all existing agents in the population are replaced by naïve individuals who inherit the problems and solutions of the previous generation. The inheritance of solutions is constrained by a loss parameter, $\epsilon$, which sets the probability of information loss in a population, $P_{\epsilon}$. Here, information loss refers to the removal of all solutions stored in a given agent's memory, with $P_{\epsilon}$ corresponding to the probability of choosing an agent to undergo this loss process (see Fig. 3 . Three probabilities were considered: $P_{\epsilon}=[0.0,0.5,1.0]$. When $P_{\epsilon}=0.0$, there is perfect inheritance of solutions in the population, whereas $P_{\epsilon}=1.0$ means there is no inheritance of solutions from one generation to the next. Finally, if $P_{\epsilon}=0.5$ approximately $50 \%$ of naïve agents will inherit the solutions of the previous generation and the other $50 \%$ of agents will not. 


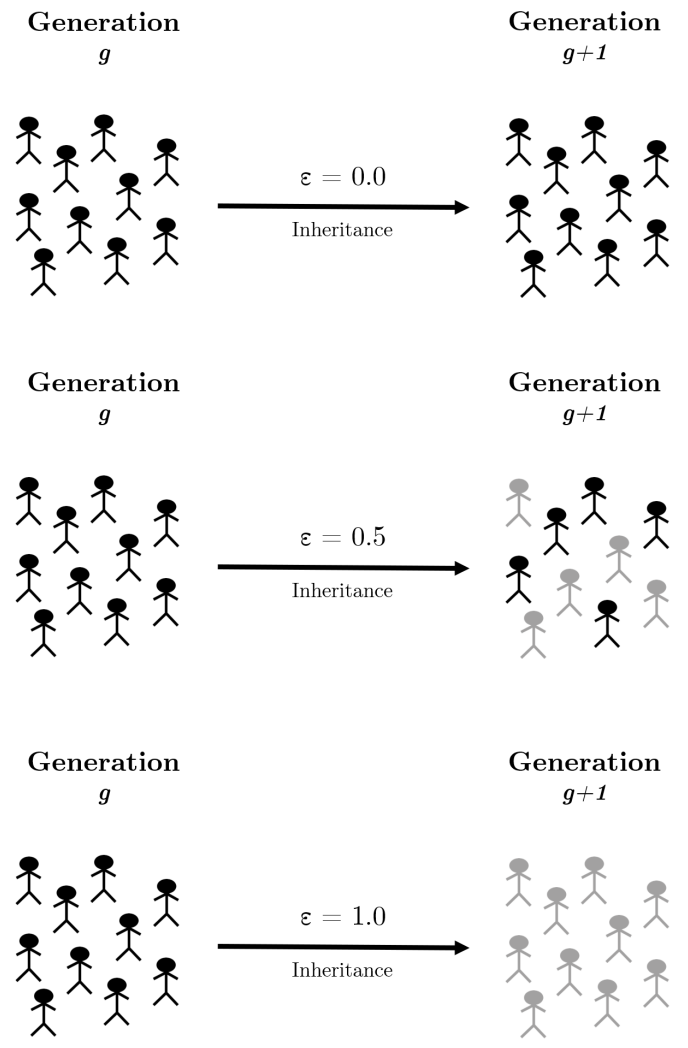

Figure 3: The level of inheritance $(\epsilon)$ from one generation to the next in a subset of agents $(n=10)$. Grey agents at generation $g+1$ denote individuals who do not inherit the solutions of the previous generation (in contrast to black agents). When $\epsilon=0.0$, all agents in the population receive the solutions of the previous generation, whereas when $\epsilon=0.5$ only $50 \%$ of agents inherit solutions and there is no inheritance when $\epsilon=1.0$.

\section{Results}

The following analyses report the problem complexity, which, for the purposes here, is operationalized as the length of longest problem in a population, $\ell(\psi)$. All else being equal, finding an optimal solution for a longer problem is more computationally intensive and thus less tractable than solving a shorter one 30 . Each simulation run takes place over 2000 generations and corresponds to parameter manipulations for both the type of innovation $(\alpha)$ and the level of information loss $(\epsilon)$. Figure 4 shows the problem complexity for $\epsilon=[0.0,0.5,1.0]$ with three types of innovation: combinatorial $(\alpha=-1.0)$, local $(\alpha=1.0)$, and both combinatorial \& local $(\alpha=0.0)$. 


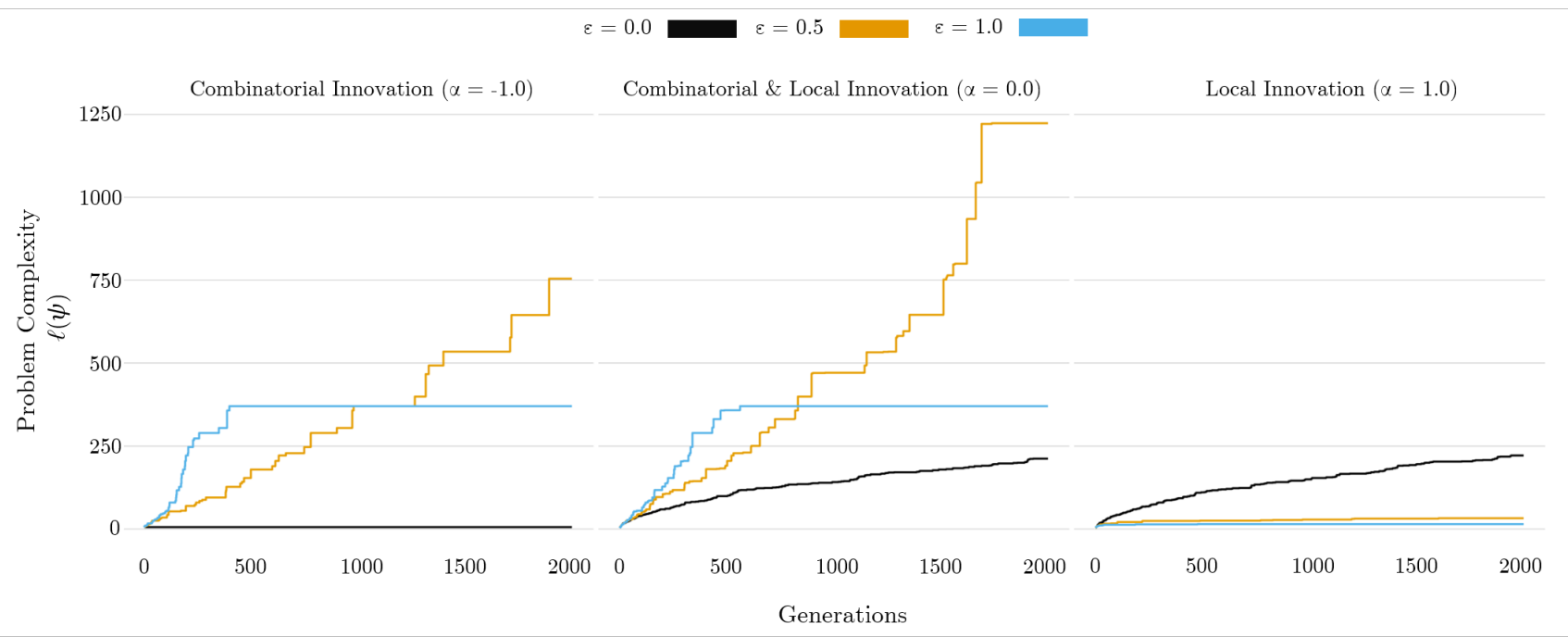

Figure 4: Single simulation runs for three levels of information loss $(\epsilon=[0.0,0.5,1.0])$ and three types of innovation $(\alpha=[-1.0,0.0,1.0])$. The $\mathrm{x}$-axis denotes the problem complexity, $\ell(\psi)$, and the y-axis corresponds to generations. Each facet refers to the type of innovation (from left to right: Combinatorial Innovation, Combinatorial \& Local Innovation, and Local Innovation) and coloured lines reflect the level of information loss (see legend).

\subsection{Minimum Information Loss $\epsilon=0.0$}

Models of cumulative cultural evolution predict that the greatest potential for growth is when information loss is low 17. This is what we find when agents only have access to local innovation $(\alpha=1.0)$ and there is perfect inheritance of information from one generation to the next $(\epsilon=0.0)$. Here, the complexity of problems continues to gradually increase over the entire runtime of the simulation, albeit subject to diminishing returns. Including combination does not substantially change the dynamics $(\alpha=0.0)$ and when local innovation is absent these combinatorial-only runs fail to exhibit any growth $(\alpha=-1.0)$.

\subsection{Moderate Information Loss $\epsilon=0.5$}

Increasing the level of information loss $(\epsilon=0.5)$, so that there is now only partial inheritance of information between generations, inhibits growth in complexity when the search dynamics are restricted to local innovation $(\alpha=1.0)$. However, in contrast to runs with perfect inheritance, having some information loss unmasks the potential of combinatorial innovation to generate growth. First, runs with combination exhibit the highest gains in complexity, especially when agents have access to both local and combinatorial modes of innovation $(\alpha=0.0)$. Second, the dynamics follow a step-wise, punctuated growth where there are periods of relative stasis followed by bursts in the dimensionality of problems. Taken together, these two points suggest combinatorial innovation is not only robust to high levels of information loss, such perturbations are necessary to unmask the potential to generate long-term growth in the complexity of problems. 


\subsection{Maximum Information Loss $\epsilon=1.0$}

Maximising information loss $(\epsilon=1.0)$, wherein there is no intergenerational inheritance of cultural information, again inhibits growth in runs where agents only have access to mechanisms of local innovation $(\alpha=1.0)$. Severing the ability to transmit information intergenerationally also creates a bound on runs with combinatorial innovation $(\alpha=[-1.0,0.0])$. In these runs, agents eventually hit a complexity ceiling, and the dynamics of growth cease as individuals cannot explore any further. The main reason for this is that the erasure of lineages essentially stops combination from discovering innovations that expand the space of possible problems. As such, the dynamics settle into a stable equilibrium, with combinatorial innovation repeatedly (re-)innovating solutions up to a certain level of problem complexity.

\section{Discussion}

Existing theoretical frameworks point to two prominent mechanistic explanations in the evolution of technology: minimizing information loss during transmission 9] and the appearance of combinatorial innovation 1, 32. Using an agent-based model, in which cultural evolutionary dynamics are treated as search processes over the space of possible problems and solutions, the current paper re-evaluated these candidate mechanisms and arrived at three main conclusions. In line with empirical observations 33 38, combinatorial innovation appears to be a game-changing mechanism in the evolution of technology: the ability to generate novel technologies by creatively combining existing ones is a fundamental pre-requisite for long-term, open-ended problem solving. However, minimizing information loss is neither necessary nor sufficient for solving increasingly difficult problems, and it is only advantageous when agents are restricted to local innovation and incremental cumulative growth. Finally, although combinatorial innovation requires some information loss to unmask its potential, some preservation of information is also needed to avoid hitting an upper-bound on complexity. Taken together, these findings help delineate the respective contributions of combinatorial innovation and cumulative culture in the evolution of technology, and point to several directions for future research.

By privileging stabilizing mechanisms of transmission, and marginalizing the importance of constraints from problem-solving, cultural evolutionary models may have overlooked the explanatory potential of combinatorial innovation. First, several models implicitly assume that the technology being copied and combined is solving the same problem for which it was originally created. However, when factoring in the application of technologies to novel problems, then imperfect transmission need not be a barrier to long-term problem-solving [18,35. The diffusion of writing systems to nonliterate groups is a good example of this: successful transmission rests on understanding and then reconstructing the core concept of writing, not accurately copying the features of other systems 39 (also see, stimulus diffusion [40]). Second, as long argued 27,41,43], biological and technological evolution differ in important ways. Although selection-like dynamics are often present in explanations of technology, and play an important role in optimization [44, combinatorial innovation is posited to be a far more pervasive force than what is observed in biology 1]. Biological processes, such as recombination and horizontal transfer, are indirect when compared to the combination of technologies and are further constrained by the need to produce a viable organism at every stage of development (what Arthur [1] refers to as a Darwinian bottleneck). Organisms, especially complex and sexually reproducing ones, cannot directly sample and repeatedly combine organs from distinct species and then repurpose them towards a new functional goal. By contrast, combination in technology can readily take two seemingly distinct and unrelated components (say, a set of wheels and a suitcase), and productively combine them to construct a novel device (e.g., a wheeled suitcase). 
The difference between model runs with and without combination makes for an important cautionary tale: explanations of bounded growth, which normally take place in restricted search spaces [45], do not necessarily generalise to situations where the search space is open-ended. In this sense, the model helps map the space of where cumulative dynamics are and are not possible, and highlights the situations in which it leads to long-term growth in complexity. If cultural evolutionary dynamics solely rely on local innovation, exploration is limited by the ability of these mechanisms to search the space of possible problems. Combinatorial innovation, and its ability to leap as opposed to creep through the search space, connects with findings that flexibility in search processes help populations avoid becoming trapped in local optima [46,47]. Specifically, the results here build on prior work by pointing to one pathway for unmasking this latent combinatorial potential: as a robust method for dealing with information loss during transmission. This robustness-enhancing effect of combination calls for a more nuanced take on the role of information loss and questions the requirement for specific stabilizing mechanisms. Information loss allows for the erasure of nonoptimal outcomes and combination provides the mechanism for rapidly searching new regions of the search space. Here, the model aligns with perspectives that argue precise copying is not necessary for long-term stability 14, 48, and similar outcomes are achievable via convergent transformation [49], inductive biases 15 or by compensating for errors in learning [18].

In previous models, mitigating information loss acts as a stabilizing mechanism, which, depending on the model, is either necessary or advantageous for cumulative dynamics. For the current model, low levels of information loss are only advantageous when combination is absent, and this advantage disappears once individuals are equipped with a combinatorial capacity. No existing theoretical framework accounts for these modulating effects of combination. Furthermore, the findings run contrary to prior expectations [17, in that increases in stability do not scale monotonically with a greater potential for solving increasingly complex problems. To some extent, this relates to other models pointing to a plurality of routes for mitigating the effects of inaccurate transmission 13, 18,50,51. However, unlike these models, combination parsimoniously brings together the stabilizing and creative aspects under a single, mechanistic explanation. All that is required is an asymmetry where there are more gains in complexity from combination than slippages due to inaccurate transmission $3,21,22$.

A key contribution here is to bridge the gap between cultural evolutionary models, which focus on the open-ended evolution of technology, with models that incorporate more sophisticated search topologies (such as those found in $N K$ landscapes [52]). Movement through this space is modelled as a search process where the goal is find efficient technologies by searching for novel solutions (optimization) or through seeking out new problems to solve (exploration). Previous models of cumulative dynamics were unable to investigate these dynamics due to the absence of rich, multidimensional search spaces 35,53]. Complicating matters is that the topology of the landscape in this model is emergent and thus continually expanding the space of problems and solutions. Arguably, this more accurately reflects what we observe in the evolution of technology, where the target of optimization is constantly moving and contingent on the discovery of novel functionalities 54 . One potential avenue for future work is to formalise the relationship between $N K$ models and the procedurally generated space used in this model. A deeper formal understanding would also be beneficial for using this framework in an experimental context. As with the $N K$ landscape, which has provided a fertile method for linking models to experiments [55,56], a similar approach could be adopted in future extensions.

A minimal set of assumptions are made with respect to the mechanisms in this model: namely, that information loss is at a system level, local innovation is restricted to single edits, and combination simply involves pasting together two existing solutions. The rationale for only looking at information loss at a system level is to make the existing results comparable with those in previous 
models 16, 17]. Relaxing this first assumption, to consider instances where individual solutions are subject to errorful and biased transmission, will potentially help resolve questions over the extent to which cultural transmission is reconstructive as opposed to preservative 57,58. The second assumption, that innovation is restricted to single edits, is meant to approximate local search processes and links with work suggesting that innovations are often introduced via limited experimentation [44 59]. Changing this innovation mechanism, to allow for greater degrees of freedom and more substantial edits, is likely to dramatically impact the search dynamics and blur the distinction between local and combinatorial innovation. Finally, while the choice of a simple combinatorial mechanism fits with existing models of cumulative growth dynamics [17,24], there is a growing body of work considering more sophisticated processes of creativity (for review, see [22]). Extending this approach, to cases where combination considers linkages between subcomponents and how these can be extracted to generate novel technologies 60], will further advance our understanding of the differences between human and non-human creative competencies.

\section{Data availability}

Code, data, and analyses are available at the following repository: https://osf.io/q8jdu

\section{Competing interests}

The author(s) declare no competing interests.

\section{References}

[1] Brian Arthur. The nature of technology: What it is and how it evolves. Simon and Schuster, 2009.

[2] Joseph Alois Schumpeter et al. Business cycles, volume 1. McGraw-Hill New York, 1939.

[3] William Fielding Ogburn. Social Change. Viking, New York, 1950.

[4] Andreas Wagner and William Rosen. Spaces of the possible: universal darwinism and the wall between technological and biological innovation. Journal of the Royal Society Interface, 11(97):20131190, 2014.

[5] Mathieu Charbonneau. Modularity and recombination in technological evolution. Philosophy \& Technology, 29(4):373-392, 2016.

[6] William F Ogburn and Dorothy Thomas. Are inventions inevitable? A note on social evolution. Political Science Quarterly, 37(1):83-98, 1922.

[7] George Basalla. The evolution of technology. Cambridge University Press, 1988.

[8] Robert Boyd and Peter J Richerson. Why culture is common, but cultural evolution is rare. Proceedings of the British Royal Society, 88:77-93, 1996.

[9] Michael Tomasello. The cultural origins of human cognition. Harvard university press, 1999.

[10] Lewis G Dean, Gill L Vale, Kevin N Laland, Emma Flynn, and Rachel L Kendal. Human cumulative culture: a comparative perspective. Biological Reviews, 89(2):284-301, 2014. 
[11] Mesoudi Alex and Thornton Alex. What is cumulative cultural evolution? Proceedings of the Royal Society B: Biological Sciences, 285(1880):20180712, June 2018.

[12] Robert Henry Thurston. A History of the Growth of the Steam-Engine, volume 24. NY: D. Appleton, 1878.

[13] Joseph Henrich. Demography and cultural evolution: how adaptive cultural processes can produce maladaptive losses - the tasmanian case. American Antiquity, 69(2):197-214, 2004.

[14] Nicolas Claidière and Dan Sperber. The role of attraction in cultural evolution. Journal of Cognition and Culture, 7(1-2):89-111, 2007.

[15] Thomas L Griffiths, Michael L Kalish, and Stephan Lewandowsky. Theoretical and empirical evidence for the impact of inductive biases on cultural evolution. Philosophical Transactions of the Royal Society B: Biological Sciences, 363(1509):3503-3514, 2008.

[16] Magnus Enquist, Pontus Strimling, Kimmo Eriksson, Kevin Laland, and Jonas Sjostrand. One cultural parent makes no culture. Animal Behaviour, 79(6):1353-1362, 2010.

[17] Hannah M Lewis and Kevin N Laland. Transmission fidelity is the key to the build-up of cumulative culture. Philosophical Transactions of the Royal Society B: Biological Sciences, 367(1599):2171-2180, 2012.

[18] Noa Truskanov and Yosef Prat. Cultural transmission in an ever-changing world: trial-and-error copying may be more robust than precise imitation. Philosophical Transactions of the Royal Society B: Biological Sciences, 373(1743):20170050, 2018.

[19] Dominik Deffner and Anne Kandler. Trait specialization, innovation, and the evolution of culture in fluctuating environments. Palgrave Communications, 5(1):1-10, 2019.

[20] W Brian Arthur and Wolfgang Polak. The evolution of technology within a simple computer model. Complexity, 11(5):23-31, 2006.

[21] Magnus Enquist, Stefano Ghirlanda, Arne Jarrick, and C-A Wachtmeister. Why does human culture increase exponentially? Theoretical population biology, 74(1):46-55, 2008.

[22] Laurel Fogarty, Nicole Creanza, and Marcus W Feldman. Cultural evolutionary perspectives on creativity and human innovation. Trends in ecology \& evolution, 30(12):736-754, 2015.

[23] Maxime Derex, Charles Perreault, and Robert Boyd. Divide and conquer: intermediate levels of population fragmentation maximize cultural accumulation. Philosophical Transactions of the Royal Society B: Biological Sciences, 373(1743):20170062, 2018.

[24] Magnus Enquist, Stefano Ghirlanda, and Kimmo Eriksson. Modelling the evolution and diversity of cumulative culture. Philosophical Transactions of the Royal Society B: Biological Sciences, 366(1563):412-423, 2011.

[25] Oren Kolodny, Nicole Creanza, and Marcus W Feldman. Evolution in leaps: the punctuated accumulation and loss of cultural innovations. Proceedings of the National Academy of Sciences, 112(49):E6762-E6769, 2015.

[26] Nicole Creanza, Oren Kolodny, and Marcus W Feldman. Greater than the sum of its parts? modelling population contact and interaction of cultural repertoires. Journal of The Royal Society Interface, 14(130):20170171, 2017. 
[27] Ricard V Solé, Sergi Valverde, Marti Rosas Casals, Stuart A Kauffman, Doyne Farmer, and Niles Eldredge. The evolutionary ecology of technological innovations. Complexity, 18(4):15-27, 2013.

[28] Michael R Garey and David S Johnson. Computers and intractability, volume 174. freeman San Francisco, 1979.

[29] Iris Van Rooij. The tractable cognition thesis. Cognitive science, 32(6):939-984, 2008.

[30] Scott Aaronson. Why Philosophers Should Care About Computational Complexity. arXiv:1108.1791 [quant-ph], August 2011. arXiv: 1108.1791.

[31] Gabriel Tarde. The Laws of Imitation. Henry Holt and Company, New York, 1903.

[32] Bernat Corominas-Murtra, Luís F Seoane, and Ricard Solé. Zipf's law, unbounded complexity and open-ended evolution. Journal of the Royal Society Interface, 15(149):20180395, 2018.

[33] Mike Steel, Wim Hordijk, and Stuart A Kauffman. Dynamics of a birth-death process based on combinatorial innovation. Journal of theoretical biology, 491:110187, 2020.

[34] Joel Mokyr. Punctuated equilibria and technological progress. The American Economic Review, 80(2):350-354, 1990.

[35] James Winters. Escaping optimization traps: the role of cultural adaptation and cultural exaptation in facilitating open-ended cumulative dynamics. Palgrave Communications, 5(1):113, 2019.

[36] Herbert A Simon. The architecture of complexity. Proceedings of the American Philosophical Society, 106(6):467-482, 1962.

[37] Vladimir Iosifovich Levenshtein. Binary codes capable of correcting deletions, insertions, and reversals. In Doklady Akademii Nauk, volume 163, pages 845-848. Russian Academy of Sciences, 1965 .

[38] H. Youn, D. Strumsky, L. M. A. Bettencourt, and J. Lobo. Invention as a combinatorial process: evidence from US patents. Journal of The Royal Society Interface, 12(106):20150272-20150272, April 2015.

[39] Olivier Morin, Piers Kelly, and James Winters. Writing, graphic codes, and asynchronous communication. Topics in cognitive science, 2018.

[40] Alfred Louis Kroeber. Stimulus diffusion. American anthropologist, 42(1):1-20, 1940.

[41] F Jacob. Evolution and tinkering. Science, 196(4295):1161-1166, 1977.

[42] Nicolas Claidière, Thomas C Scott-Phillips, and Dan Sperber. How darwinian is cultural evolution? Philosophical Transactions of the Royal Society B: Biological Sciences, 369(1642):20130368, 2014.

[43] Daniel Nettle. Selection, adaptation, inheritance and design in human culture: the view from the price equation. Philosophical Transactions of the Royal Society B, 375(1797):20190358, 2020 . 
[44] Peter J. Richerson and Robert Boyd. Not By Genes Alone: How Culture Transformed Human Evolution. University of Chicago Press, June 2005.

[45] Acerbi Alberto, Tennie Claudio, and Mesoudi Alex. Social learning solves the problem of narrowpeaked search landscapes: experimental evidence in humans. Royal Society Open Science, 3(9):160215, 2016.

[46] Christina Fang, Jeho Lee, and Melissa A Schilling. Balancing exploration and exploitation through structural design: The isolation of subgroups and organizational learning. Organization Science, 21(3):625-642, 2010.

[47] Matthew Spike, Kevin Stadler, Simon Kirby, and Kenny Smith. Minimal requirements for the emergence of learned signaling. Cognitive science, 41(3):623-658, 2017.

[48] Olivier Morin. How traditions live and die. Oxford University Press, 2016.

[49] Nicolas Claidière, Gameli Kodjo-kuma Amedon, Jean-Baptiste André, Simon Kirby, Kenny Smith, Dan Sperber, and Joël Fagot. Convergent transformation and selection in cultural evolution. Evolution and Human Behavior, 39(2):191-202, 2018.

[50] Adam Powell, Stephen Shennan, and Mark G Thomas. Late pleistocene demography and the appearance of modern human behavior. Science, 324(5932):1298-1301, 2009.

[51] Mathieu Charbonneau. Understanding cultural fidelity. The British Journal for the Philosophy of Science, 2018.

[52] Stuart Kauffman and Simon Levin. Towards a general theory of adaptive walks on rugged landscapes. Journal of theoretical Biology, 128(1):11-45, 1987.

[53] Masaki Suyama and Kosuke Sato. Property of innovation under different group size in an openended fitness landscape: Demonstration through evolutionary logical circuits. arXiv preprint arXiv:2002.00593, 2020.

[54] Teppo Felin, Stuart Kauffman, Roger Koppl, and Giuseppe Longo. Economic opportunity and evolution: Beyond landscapes and bounded rationality. Strategic Entrepreneurship Journal, 8(4):269-282, 2014.

[55] David Lazer and Allan Friedman. The network structure of exploration and exploitation. Administrative science quarterly, 52(4):667-694, 2007.

[56] Kyanoush Seyed Yahosseini and Mehdi Moussaïd. Comparing groups of independent solvers and transmission chains as methods for collective problem-solving. Scientific reports, 10(1):1-9, 2020 .

[57] Dan Sperber. Explaining culture: A naturalistic approach. Cambridge, MA: Cambridge, 1996.

[58] Alberto Acerbi, Mathieu Charbonneau, Helena Miton, and Thom Scott-Phillips. Cultural stability without copying. 2019.

[59] Tennie Claudio, Call Josep, and Tomasello Michael. Ratcheting up the ratchet: on the evolution of cumulative culture. Philosophical Transactions of the Royal Society B: Biological Sciences, 364(1528):2405-2415, August 2009. 
[60] D Justin Yeh, Laurel Fogarty, and Anne Kandler. Cultural linkage: the influence of package transmission on cultural dynamics. Proceedings of the Royal Society B, 286(1916):20191951, 2019 . 